\title{
Outcomes and Complications of Sutured Scleral-Fixated Foldable Intraocular Lens Implantation: A Retrospective Study of 5-Year Follow-Up
}

\author{
Ting Yu $\mathbb{D},{ }^{1}$ Mengting Yu, ${ }^{1}$ Wenjie Wu $\mathbb{D}^{1,2}$ Xinna Wu, ${ }^{1}$ Suzhen Xiao, ${ }^{1}$ Jialiang Mao, ${ }^{3}$ \\ and Yanling Wang ${ }^{4}$ \\ ${ }^{1}$ Ophthalmology Department, Provincial Clinical Medical College of Fujian Medical University, Fuzhou 350001, China \\ ${ }^{2}$ Ophthalmology Department, Fujian Provincial Hospital, Fuzhou 350001, China \\ ${ }^{3}$ Ophthalmology Department, Guangze County Hospital, Nanping 353000, China \\ ${ }^{4}$ Ophthalmology Department, Xiapu Funing County Hospital, Ningde 352000, China
}

Correspondence should be addressed to Wenjie Wu; wenjie_wu@foxmail.com

Received 8 February 2021; Revised 11 April 2021; Accepted 8 July 2021; Published 17 July 2021

Academic Editor: Michele Figus

Copyright $(92021$ Ting Yu et al. This is an open access article distributed under the Creative Commons Attribution License, which permits unrestricted use, distribution, and reproduction in any medium, provided the original work is properly cited.

Purpose. To evaluate long-term outcomes and complications of sutured scleral-fixated foldable intraocular lens (IOL) implantation. Design. Retrospective study. Methods. Patients who underwent sutured scleral-fixated foldable IOL implantation using 10-0 polypropylene suture were followed up for at least 5 years at one Chinese tertiary hospital and two primary hospitals. Results. 52 eyes among 48 patients ( 35 male and 13 female) were evaluated. The mean age (years) was $50.27 \pm 20.08$ (range: 6 to 81 ). The mean postoperative follow-up time (months) was $79.70 \pm 18.84$ (range: 60 to 121). The mean best-corrected visual acuity (BCVA) improved from $0.83 \pm 0.69$ logarithm of the minimum angle of resolution (logMAR) at baseline to $0.50 \pm 0.45 \log$ MAR at the last follow-up visit. There was improved or unchanged BCVA in 44 eyes (84.62\%) and reduced BCVA in 8 eyes (15.38\%). Mild intraoperative intravitreal hemorrhage was observed in 3 eyes $(5.77 \%)$. Early postoperative complications included transient elevated intraocular pressure (IOP) in 5 eyes $(9.62 \%)$ and hypotony in 1 eye (1.92\%). Secondary epimacular membrane occurred in 5 eyes $(9.62 \%)$ and retinal detachment (RD; 3 years postsurgery), subconjunctival suture knot exposure ( 5 years postsurgery), and persistent elevated IOP (in a GRAVES patient) occurred in 1 eye $(1.92 \%)$ each. No suture erosion or breakage nor IOL dislocation was observed. No visually threatening IOL tilt or decentration was reported in any patient. Conclusion. Sutured scleral-fixated foldable IOL implantation demonstrated satisfactory long-term outcomes and rare suture-related complications. This technology was safe and did not require complicated equipment and is of considerable interest in the setting of aphakia without adequate capsule support.

\section{Introduction}

There are several mainstream surgical approaches to correct aphakia without adequate capsular support. Current choices include implantation of an iris-fixated intraocular lens (IOL) (pre- or retropupillary), sutureless intrascleral posterior chamber IOL fixation, and scleral-fixated IOL [1-8]. Although angle-supported anterior chamber IOL (AC-IOL) was adopted in 1952, its use today is limited due to high long-term risks of bullous keratopathy and glaucoma $[5,6]$. Scleral-fixated IOL (SF-IOL), including sutureless and sutured fixated IOL (SSF-IOL), continues to gain acceptance among surgeons $[4,9]$.

Sutureless ciliary sulcus-fixation technique, as proposed by Gabor and Pavlidis [2] in 2007, attempted to avoid suture-related complications. However, most reports of implantation of sutureless intrascleral posterior chamber IOL had short (1-55 months) follow-up periods $[4,9-11]$ and subconjunctival IOL haptic exposure, IOL dislocation (as early as 1 day postsurgery), and pupil capture have been reported $[10,12,13]$. Additionally, the need for complicated equipment/instruments and specialized surgical skills 
challenge the use of such techniques in developing and underdeveloped countries, especially in primary hospitals and eye centers.

Considering these obstacles, SSF-IOL implantation remains an effective procedure. Its long-term outcomes and safety profile have been widely reported [14-24]. The most concerning late complication was IOL dislocation due to suture breakage, occurring on average at approximately 50 months postsurgery, with differences between studies and ethnic groups [15-17]. For example, suture breakage in Caucasians varied from $0 \%$ to $57.69 \%$ [21-23, 25-28] at 12-294 months of follow-up, whereas in more darkly pigmented groups such as Asians and Africans, it varied from $0 \%$ to $4.65 \%[14,19,20,29-31]$ at $12-180$ months of followup. Other reported adverse events (AEs) included lens tilt, suprachoroidal or vitreous hemorrhage, retinal detachment (RD), and endophthalmitis, which varied among studies $[14,15,18,32]$.

Several studies have retrospectively evaluated long-term outcomes and complications of SSF-IOL implantation in Asian and African patients [14, 19, 20, 29-31]. Of note, reports by Kim et al. [20] and Yang and Chao [19] were based on relatively small cohorts (15 and 29 cases, respectively), and those by Zhao et al. [24] and Rogers et al. [29] were based on follow-up periods from 6 to 99 months and from 0 to 54 months, respectively. In one large retrospective review by Luk et al. [14] with follow-up ranging from 12 to 180 months, procedures were performed by four different surgeons. The study reviewed postoperative AEs, but lacked detail regarding time of occurrence. The purpose of our study was to evaluate the long-term safety, efficacy, and clinical outcomes of SSF-IOL implantation over a 5-year period in China. Special emphasis was placed on AEs including suture-related complications, IOL dislocation, hypotony, elevated intraocular pressure (IOP), and RD.

\section{Materials and Methods}

SSF-IOL implantation was performed by the same surgeon at three sites: Fujian Provincial Hospital (tertiary referral site) and Guangze County Hospital and Xiapu County Hospital (primary hospital sites). The study was conducted in compliance with the guidelines of the Declaration of Helsinki and was approved by the ethics committees of all participating hospitals.

To evaluate long-term outcomes, we conducted a retrospective investigation of patients with at least 5 years of follow-up data between December 2009 and November 2015. In total, 101 patients (27 female and 74 male) underwent surgery during this period. We excluded 6 patients who had incomplete medical records. Among the remaining 95 patients, 46 were excluded for the following: 8 died, 4 were unable to visit clinic due to disability, and 34 were lost to follow-up. Finally, we studied 52 eyes ( 3 in Guangze County Hospital, 2 in Xiapu County Hospital, and 47 in Fujian Provincial Hospital) among 48 patients (35 male and 13 female). Preoperative data included demographics, bestcorrected visual acuity (BCVA), IOP, lens status, previous surgeries, preexisting ocular pathologies, and history of ocular trauma. Axial length (AL) was measured by using a partial coherence interferometer (IOL Master, Carl Zeiss AG, Jena, Germany) or A-scan ultrasound biometry (AL4000 Pachymeter, Japan) prior to surgery. The refractive power of the IOL was calculated using the SRK/T formula for AL between 21 and $26 \mathrm{~mm}$ and the Haigis formula for $\mathrm{AL}$ exceeding this interval.

Visual outcomes were measured by the distance Snellen chart preoperatively and at the last clinic visit. Pre- and postoperative BCVA were measured. Snellen acuity was converted to the logarithm of minimal angle of resolution ( $\log$ MAR) VA for analysis. We used a logMAR VA of 2 and 3 , respectively, to represent counting fingers and hand movement vision [33]. The spherical equivalent (SE) value was calculated as the sum of the spherical power with half of the cylindrical power. The refractive prediction error (RPE) was calculated by subtracting the estimated preoperative SE from the postoperative SE. Considering that developing AL might interfere with RPE, we excluded subjects who were less than 18 years of age.

Early postoperative complications were defined as AEs occurring within 1 month postsurgery. Any AE occurring after 1 month was considered a late complication. We observed the 10-0 polypropylene suture/suture knot with a slitlamp and, when the suture/suture knot was visible under the conjunctival, we used bulbar conjunctival fluoresce staining to determine whether the suture/suture knot was exposed beyond the conjunctival epithelium.

2.1. Surgical Technique. Procedures were performed by the same surgeon using a similar technique (video and supplemental digital content are available at https://drive. google.com/file/d/1Ff5wC1Uf1pqohpw1j-S3kiiTwfMbbjhp/ view? usp=sharing). Retrobulbar or general anesthesia (2 children) was administered. A three-piece (ZA9003, Tecnis, AMO) or one-piece (ZCB00, Tecnis, AMO) IOL was implanted. Two opposing limbus-based triangle scleral flaps were prepared $1.5 \mathrm{~mm}$ from the limbus at 4-5 o'clock and 1011 o'clock in 48 eyes. In 4 eyes with a partial residual capsule, a single-suture scleral fixation was used to position the IOL haptic at the absent capsular position. The ab externo technique was used as described below. A straight needle carrying a 10-0 polypropylene suture was inserted into the posterior chamber through one scleral flap. A 26-gauge needle was then inserted through the opposite scleral flap to pull the straight needle out of the eye within its barrel. Then, a 2.4 or $3.0 \mathrm{~mm}$ corneal incision was made at 9 o'clock and the suture was pulled out through the corneal incision and cut off. A foldable IOL was loaded in the injector, part of the foregoing (leading) haptic was pushed out of the cartridge, and the suture was tied with at least 5 knots to the maximum radian of the IOL haptic to prevent suture slippage (Figure 1(a)). For the three-piece IOL (38 subjects), the haptic end was heated to create a mushroom-shaped flange of about $0.16 \mathrm{~mm}$. For the one-piece IOL (10 subjects), the end was not heated, but the haptic was slightly depressed by the suture to reduce suture movement. Then, after tying the suture to the leading haptic, the plunger was withdrawn 

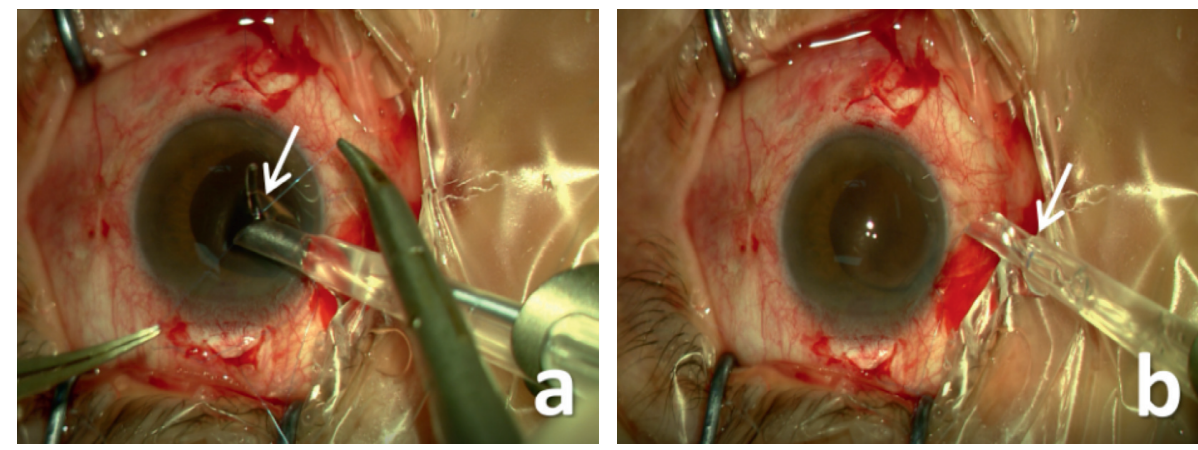

FIgURE 1: Sutured scleral-fixated implantation with a one-piece foldable IOL. (a) The suture was tied to the maximum radian of the IOL haptic with at least 5 knots. (b) After withdrawing the plunger, the haptic drew back into the cartridge.

(Figure 1(b)), so that the IOL could be implanted into the posterior chamber through a smaller $(2.4 \mathrm{~mm})$ corneal incision. Subsequently, the cartridge was inserted into the corneal incision and the posterior (trailing) haptic was left outside the incision for suture fixation as aforementioned. Then, the sutured posterior haptic was carefully inserted into the posterior chamber through the corneal incision using microforceps. After tensioning the sutures, the IOL was placed in a central position and the corneal incision was closed using a 10-0 nylon suture followed by suturing of the scleral flaps and conjunctiva. For secondary lens implantation in eyes without coexistent vitreoretinal disorders, no vitrectomy was performed and infusion or AC maintainer was not applied. For those with minor vitreous incarceration in the pupil area/corneal incision, a scissors was used to excise it. In 4 eyes with prior pars plana vitrectomy (PPV), vitreous infusion was used to maintain intraocular stability. In 5 eyes with RD, PPV with SSF-IOL implantation was performed at the same time.

2.2. Statistical Analysis. Means and standard deviations (SDs) of the quantitative variables were calculated. A paired $t$-test was used to detect differences in quantitative variables when data obeyed normal distribution; otherwise, the Wilcoxon matched-pairs signed ranks sum test was used. Differences were considered statistically significant if the $P$ value was $<0.05$. All calculations were performed using SPSS software (version 24, SPSS, Inc.).

\section{Results}

In the present study, 52 eyes among 48 patients ( 35 male and 13 female) were evaluated. The characteristics of the study population are shown in Table 1 . The mean age was $50.27 \pm 20.08$ (range: $6-81$ ) years. The mean follow-up time was $79.7 \pm 18.84$ (range: $60-121$ ) months.

3.1. Refractive and Visual Outcomes. The mean preoperative BCVA was $0.83 \pm 0.69 \log$ MAR; the mean postoperative BCVA was $0.50 \pm 0.45 \log$ MAR at the last follow-up $p<0.05$

. In 44 eyes $(84.62 \%)$, BCVA improved or remained unchanged; in 8 eyes (7.2\%), it worsened (Figure 2). Reasons for BCVA decline included secondary epimacular membrane ( 3 eyes), progressive epimacular membrane ( 1 eye), optic atrophy (1 eye), and retinitis pigmentosa (3 eyes).

At the last follow-up visit, the mean SE was $-1.00 \pm 1.74$ diopters, and the mean RPE was $-0.67 \pm 1.31$ diopters. RPEs in 32 eyes $(61.54 \%)$ were within 1.00 diopters and in 15 eyes (28.85\%) were within 2.00 diopters. In 2 eyes (3.85\%), RPE exceeded 4.00 diopters; both had high axial myopia $(27.31 \mathrm{~mm}$ and $29.08 \mathrm{~mm}$ ) and posterior scleral staphyloma, and their ALs had been measured by A-ultrasound in a county hospital, which might explain the unexpectedly large postoperative RPEs.

\subsection{Complications}

3.2.1. Intraoperative. In 3 eyes (2 patients), vitreous hemorrhage occurred in association with passing the 10-0 polypropylene suture through the sclera $1.5 \mathrm{~mm}$ posterior to the limbus. In 1 eye, the hemorrhage appeared to arise from extraocular blood wicked into the eye through the puncture; it was mild and stopped after hemostasis. In the other 2 eyes, the patient had Marfan syndrome and took an oral anticoagulant (Warfarin) after cardiac surgery; the hemorrhage may have come from the ciliary body. Surgery proceeded and the vitreous hemorrhage resolved within 2 weeks. There was no other case of intraoperative complications such as choroidal detachment or suprachoroidal hemorrhage.

3.2.2. Postoperative. As shown in Table 2, early complications included transient elevated IOP in 5 eyes $(9.62 \%)$ and hypotony in 1 eye $(1.92 \%)$ that had previous PPV surgery. Late complications included retinal detachment, subconjunctival suture knot exposure, and persistent elevated IOP in 1 eye $(1.92 \%)$ each; the latter occurred in an eye with GRAVES. Epimacular membrane occurred in 5 eyes $(9.62 \%)$. In the eye with $\mathrm{RD}$, the $1 / 5 \mathrm{PD}$ round hole was located near the $9: 30$ o'clock 20G trocar position, away from the suture-fixation position. We observed few complications associated with sutures; in most patients, suture knots could be seen in the subconjunctiva (Figure 3(a)), but staining was negative (Figure 3(b)). Only in 1 eye $(1.92 \%)$, one suture knot exposure occurred 5 years after surgery with positive staining, but no suture knot erosion was observed (Figure 3(c)). We performed a conjunctival separation and 
TABle 1: Patient characteristics.

Parameter

Male : female

Age (years, mean $\pm \mathrm{SD}$ )

$35: 13$

Follow-up time (months, mean $\pm \mathrm{SD}$ )

$50.27 \pm 20.08$

Right eye : left eye

$79.7 \pm 18.84$

Preoperative comorbidities, $n$ (\%)

Trauma

$27: 25$

Postglaucoma surgery

Glaucoma with vitreous incarceration

History of retinal detachment

$2(3.85 \%)$

Marfan syndrome

$4(7.69 \%)$

$5(9.62 \%)$

Myopic degeneration

$3(5.77 \%)$

$2(3.85 \%)$

Surgical indication, $n$ (\%)

Aphakia after complicated cataract surgery

Aphakia after traumatic cataract surgery

Aphakia resulting from previous pars plana vitrectomy (PPV) for RD or trauma

Dislocated crystalline lens

Dislocated IOL

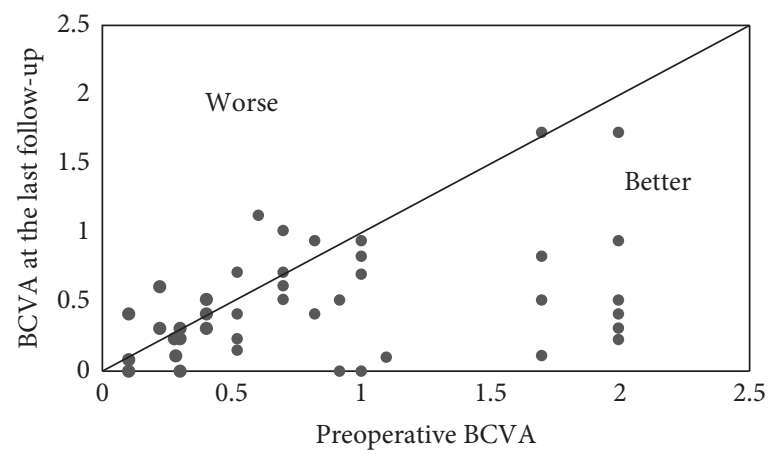

FIGURE 2: Scattergram of BCVA in 52 eyes that underwent SSF-IOL implantation. BCVA is represented in logMAR values. BCVA: bestcorrected visual acuity; SSF-IOL: sutured scleral-fixated intraocular lens.

TABLE 2: Distribution and management of postoperative complications in eyes undergoing SSF-IOL.

\begin{tabular}{lcccc}
\hline & & \multicolumn{3}{c}{ Complication } \\
Early $(\leq 1$ month) & $n(\%)$ & Mean duration postsurgery & Range & Management \\
\hline Increased IOP & $5(9.62)$ & $1.6 \pm 0.8$ days & $1-3$ days & Medical management \\
Transient vitreous hemorrhage & $3(5.77)$ & 1 day & 1 day & Medical management; resolved within 2 weeks \\
Transient hypotony & $1(1.92)$ & 1 day & 1 day & Suture the incision \\
\hline Late $(>1$ month) & $n(\%)$ & - & - & - \\
Suture knot exposure & $1(1.92)$ & 5 years & 5 years & Surgical management \\
Retinal detachment & $1(1.92)$ & 3 years & years & Surgical management \\
Increased IOP & $1(1.92)$ & 6 years & 6 years & Medical management \\
\hline
\end{tabular}
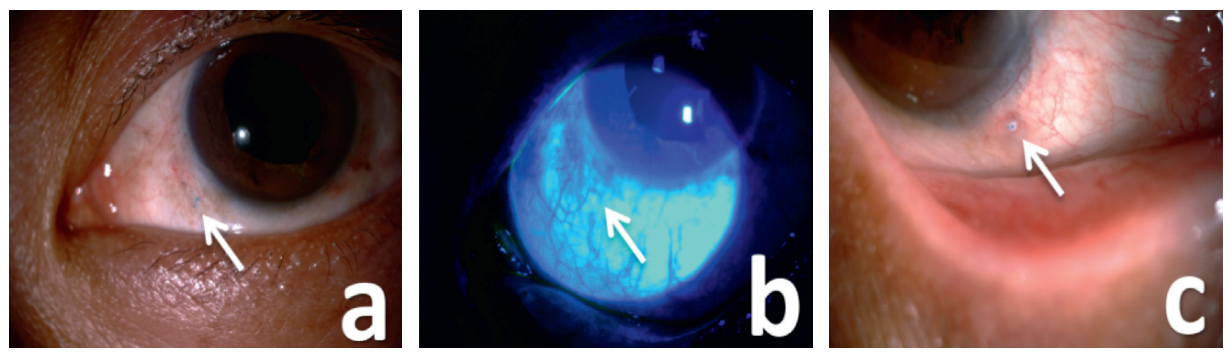

FIgURE 3: Slit-lamp microscopy images of the suture knot. (a) Suture knot visible under the conjunctiva. (b) Negative bulbar conjunctival fluoresce staining. (c) Suture exposure 5 years postsurgery. 
coverage to rescue it. No optical disturbing IOL tilt or decentration was observed (Figure 4).

It is notable that no suture breakage or IOL displacement was observed during follow-up of any patient in this study.

\section{Discussion}

Compared to iris-fixed IOL and AC-IOL, SF-IOL is superior in protecting the integrity of the anterior chamber, minimizing uveal contact, and independence of the presence of iris tissue $[3,8]$. However, due to the need for vitrectomy equipment and specialized surgical skill, sutureless SF-IOL is not likely to be widely used in primary hospitals and eye centers, especially in undeveloped/developing countries. There are few reports of sutureless SF-IOL with extended follow-up [4, 9-11]. In contrast, the SSF-IOL is a time-tested method initially described in 1986 by Malbran et al. [34]. Its long-term track record and independence from vitrectomy equipment has made it a primary implant technique worldwide in patients without sufficient capsular support [14-24].

We report the long-term outcomes of SSF-IOL implantation via a small $(\leq 3 \mathrm{~mm})$ corneal incision using 10-0 polypropylene suture. Included are cases performed at the beginning of the learning phase and those performed in two primary hospitals. Short- and long-term complications were infrequent and clinical outcomes were favorable. The SSF foldable IOL technique is less traumatic as fewer manipulations inside the eyeball are needed: suture presetting, suture out-pulling, and IOL inserting, with puncture performed when the eyeball was intact and the other procedures were finished under a small $(\leq 3 \mathrm{~mm})$ incision. For eyes having secondary lens implantation without coexistent vitreoretinal disorders, 38 eyes $(73.08 \%)$ at the tertiary hospital and 5 eyes $(9.62 \%)$ at the primary county hospitals did not require vitrectomy. When there was minor vitreous incarceration in the pupil area/corneal incision, the vitreous can be excised using scissors and then using a miotic agent. The use of a thinner 10-0 polypropylene suture preset through the ciliary sulcus with ab externo technique and smaller suture puncture were associated with minimal vitreous fluid outflow and only minor change in IOP. Little disturbance of the intraocular environment helps maintain the integrity and stability of the eyeball, such that infusion or AC maintainer is not needed.

Short-term complications included vitreous hemorrhage (5.77\%), transient elevated IOP (9.62\%), and hypotony $(1.92 \%)$. Mild transient intravitreal hemorrhage was observed in 3 eyes ( 2 patients); one of these patients had Marfan syndrome and the AE might be attributable to Warfarin after cardiac surgery. In these patients, hemorrhage resolved within 2 weeks. In another case, extraocular blood may have wicked into the eye through the puncture; however, it was mild and resolved with well hemostasis. The incidence of vitreous hemorrhage was comparable to previous reports by Yeung et al. [35] and Zhao et al. [24] (5\% and $6.6 \%$, respectively). Transient elevated IOP was observed within 3 days postoperatively in 5 eyes. All eyes were stabilized with topical medication within a few days
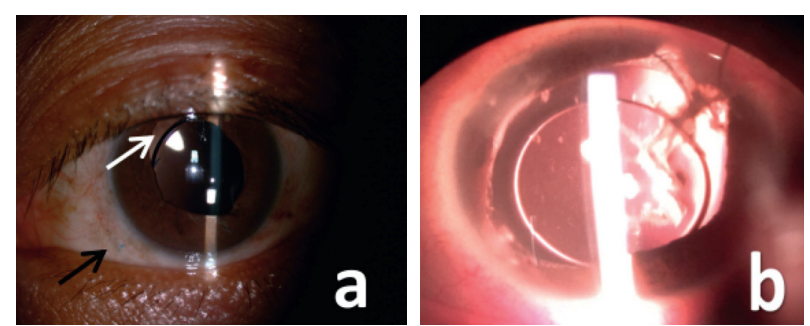

FIGURE 4: Slit-lamp microscopy images of well-centered IOLs. (a) Stable IOL position 97 months postsurgery. The black arrow points to the suture knot seen under conjunctiva. The white arrow points to the iris defect and pupil distortion due to previous trauma. (b) Well-placed IOL in a traumatic eye with atrophic iris 120 months postsurgery.

postsurgery. The supposed reasons of ocular hypertension included postoperative inflammation, retained viscoelastic agents, and temporary dysfunction of the trabecular meshwork [25]. Our study implanted a foldable SSF-IOL through a small corneal incision, using only a small amount of viscoelastic agents with minimal manipulation and vitreous disturbance. Even in the 4 cases with previous ocular hypertension resulting from vitreous incarcerate in the pupil area, IOP returned to normal after excising vitreous and implanting IOL. The ocular hypertension rate in our study was significantly lower than those in SSF-IOL studies with methyl methacrylate (PMMA) material (range: 10\%-44\%) $[16,25-27]$ and comparable to others with foldable material (range: 7\%-11.5\%) [20,36, 37]. Hypotony caused by incision leakage was observed in 1 eye post-PPV 1 day postsurgery. Thereafter, the main incision was sutured at the end of surgery to prevent postoperative hypotony, especially in vitrectomized eyes, and no further hypotony occurred. Consequently, the hypotony rate in this study is in stark contrast to studies (range: $4.3 \%-9.4 \%$ ) [16, 24, 37].

IOL dislocation due to suture breakage was a late complication and is considered by surgeons to be the greatest challenge of this technique. The incidence of this complication, typically observed $3-5$ years postprocedure [14-24], is estimated to be $0 \%-57.69 \%$ in Caucasians [23-28] and $0 \%$ to $4.65 \%$ in Asians and Africans $[14,19,20,29-31]$. In our study, there was no suture breakage with a mean follow-up of 79.7 months. The role of pigment is unclear and requires further randomized, multicenter prospective study. Furthermore, according to some reports, the risk of postoperative suture breakage was greater in younger patients (range: $12 \%-24 \%$ ) $[17,18]$; however, in our study, there was no breakage in 13 patients who are $<40$ years old. An Indian report by Bhojwani et al. [31] including 12 children (under 16 years old) also found no suture breakage. Thus, we consider suture breakage in younger Asian patients is worth further investigation.

Although 9-0 polypropylene suture has been widely used recently $[32,38,39]$, our study revealed no suture breakage, perhaps demonstrating the stability of $10-0$ polypropylene scleral fixation sutures. This result was consistent with Luk et al. [14], with a mean follow-up of 73.4 months. We consider 10-0 polypropylene suture to have some advantages. First, a 10-0 polypropylene suture knot is smaller than 
a 9-0 knot and may produce fewer complications such as scleral atrophy above the knot and erosion of the stiff cutting ends $[14,26]$. In most patients in our study, suture knots were seen in the subconjunctiva, but bulbar conjunctival fluorescein staining was negative (Figures 3(a) and 3(b)). Among 100 suture knots in 52 eyes, only 1 exposure was observed 5 years postsurgery with positive staining (Figure 3(c)), which was rescued by conjunctival separation and coverage. Smaller knots and softer thread arms of the 10-0 suture might produce less suture exposure and erosion. Second, when presetting sutures, the thicker and stiffer texture of a 9-0 or 8-0 suture may result in larger puncture allowing more intravitreous fluid leakage. What is more, there are several reports of a 10-0 polypropylene suture knotless $Z$-suture technique that demonstrate promising clinical results with follow-up from 6 to 135 months and that might further enhance SSF-IOL implantation [22, 23].

Other long-term AEs, including RD and significant lens tilt or decentration, were also infrequent in our study. $\mathrm{RD}$ was seen in 1 eye $(1.92 \%) 3$ years postsurgery with the retinal hole located away from the suture-fixation position (11 o'clock). We ascribe the detachment to the vitreous traction around the trocar rather than suture-fixation surgery. The retina was reattached successfully with no negative sequelae. Lens tilt and decentration are well-documented complications in SSF-IOL implantation. Durak et al. [40] reported a $16.7 \%$ rate of lens tilt or decentration. Lens tilt develops due to asymmetric suture placement, and decentration occurs due to the asymmetric attachment of suture haptics on the scleral bed, loose suture, suture breakage, or other causes [40]. We identified no significant IOL decentration or tilt in our study at a mean follow-up of 79.7 months.

After IOL implantation, all patients had improved vision. In 44 eyes $(84.62 \%)$, BCVA improved or remained unchanged and worsened in 8 eyes (7.2\%) at the last followup visit. Most previous studies showed increased or unchanged BCVA after SF-IOL implantation in 86.2\%-92.8\% of cases [24, 32, 35]. Visual outcomes in our study were impressive and comparable to the previous study with a high rate of BCVA improvement, demonstrating long-term stability of SSF foldable IOL. Those eyes with worsening BCVA over the follow-up period resulted from epimacular membrane, optic atrophy, and retinitis pigmentosa, which were inextricable. At the last follow-up, RPEs in 32 eyes (61.54\%) were within 1.00 diopters and in 15 eyes (28.85\%) were within 2.00 diopters. There is no consensus on the target spherical equivalent when implanting an SF-IOL. Because sulcus-fixated IOL is located more anteriorly than in-the-bag fixation, postoperative refraction can lead to a myopic shift from the predicted value. In our study, an unexpected myopic shift occurred in 2 eyes ( -4.22 diopters and -5.74 diopters), both at county hospitals, with high axial myopia $(27.31 \mathrm{~mm}$ and $29.08 \mathrm{~mm}$ ) and posterior scleral staphyloma. Due to the lack of optical biometry there, ALs were measured by A-ultrasound, which could explain the large postoperative spherical equivalent deviation. Therefore, we strongly recommended taking careful repeated measures of AL and applying precise optical biometry in patients with high axial myopia and posterior scleral staphyloma.

There were some limitations to this study, primarily the retrospective design and the absence of a control group. However, its greatest strength was the long ( $>5$ years) follow-up period. Another limitation was substantial (49\%) loss to follow-up, which might introduce selection bias. Nevertheless, in our study, patient data were collected retrospectively for more than 5 years, and the loss to follow-up was a limitation inherent to the long-term retrospective design. Anterior segment optical coherence tomography or Scheimpflug images were not reported as a more accurate indication of IOL tilt or decentration; therefore, large, prospective, and long-term randomized clinical trials are required to further substantiate the therapeutic benefits demonstrated in this study.

\section{Conclusions}

Our study validated the beneficial long-term outcomes of SSF foldable IOL implantation in China via a small $(\leq 3 \mathrm{~mm})$ corneal incision using 10-0 polypropylene suture. This technology was safe, easy to master, and easily replicated. Both short- and long-term AEs (particularly suture-related complications) were rare and long-term visual outcomes were stable. Free from relying on complicated resources, such as expensive equipment and vitrectomy skills, SSF-IOL technology may be especially useful in primary hospitals and eye centers, and in underdeveloped or developing settings.

\section{Data Availability}

The dataset used for analyses may be requested from the corresponding author for use in scholarly work related to the field.

\section{Disclosure}

The sponsor or funding organization had no role in the design or conduct of this research.

\section{Conflicts of Interest}

The authors declare no conflicts of interest regarding the publication of this paper.

\section{Acknowledgments}

Publication of this article was supported by the Medical Innovation Project of Fujian, China (2020CXA005). The authors thank Caron Modeas for editorial assistance.

\section{Supplementary Materials}

The supplementary materials are two videos which describe the surgical procedure in detail. (Supplementary Materials)

\section{References}

[1] R. T. Peralba, D. Lamas-Francis, T. Sarandeses-Diez, L. Martínez-Pérez, and T. Rodríguez-Ares, "Iris-claw 
intraocular lens for aphakia: can location influence the final outcomes?" Journal of Cataract and Refractive Surgery, vol. 44, no. 7, pp. 818-826, 2018.

[2] S. G. B. Gabor and M. M. Pavlidis, "Sutureless intrascleral posterior chamber intraocular lens fixation," Journal of Cataract and Refractive Surgery, vol. 33, no. 11, pp. 1851-1854, 2007.

[3] M. D. Sindal, C. P. Nakhwa, and S. Sengupta, "Comparison of sutured versus sutureless scleral-fixated intraocular lenses," Journal of Cataract and Refractive Surgery, vol. 42, no. 1, pp. 27-34, 2016.

[4] J. Nehme, M. Sahyoun, M. Saad et al., "Secondary intraocular lens implantation with absence of capsular support: scleral versus iris fixation," Journal Français d'Ophtalmologie, vol. 41, no. 7, pp. 630-636, 2018.

[5] K. Droutsas, A. Lazaridis, G. Kymionis et al., "Endothelial keratoplasty in eyes with a retained angle-supported intraocular lens," International Ophthalmology, vol. 39, no. 5, pp. 1027-1035, 2019.

[6] R. Oltulu, I. Erşan, G. Şatırtav, M. Donbaloglu, H. Kerimoglu, and A. Ozkagnici, "Intraocular lens explantation or exchange: indications, postoperative interventions, and outcomes," Arquivos Brasileiros de Oftalmologia, vol. 78, no. 3, pp. 154157, 2015.

[7] M. Schallenberg, D. Dekowski, A. Hahn, T. Laube, K. P. Steuhl, and D. Meller, "Aphakia correction with retropupillary fixated iris-claw lens (Artisan)-long-term results," Clinical Ophthalmology, vol. 8, pp. 137-141, 2014.

[8] N. Madhivanan, S. Sengupta, M. Sindal, P. Nivean, M. Kumar, and M. Ariga, "Comparative analysis of retropupillary iris claw versus scleral-fixated intraocular lens in the management of post-cataract aphakia," Indian Journal of Ophthalmology, vol. 67, no. 1, pp. 59-63, 2019.

[9] A. C. Bonnell, D. Mantopoulos, H. M. Wheatley, and J. L. Prenner, "Surgical technique for sutureless intrascleral fixation of a 3-piece intraocular lens using a 30-gauge needle," Retina, vol. 39, no. 1, pp. S13-S15, 2019.

[10] M. P. Czajka, A. Frajdenberg, M. Stopa, T. Pabin, B. Johansson, and G. Jakobsson, "Sutureless intrascleral fixation using different three-piece posterior chamber intraocular lenses: a literature review of surgical techniques in cases of insufficient capsular support and a retrospective multicentre study," Acta Ophthalmologica, vol. 98, no. 3, pp. 224-236, 2020.

[11] B. Todorich, M. S. Stem, K. Kooragayala et al., "Structural analysis and comprehensive surgical outcomes of the sutureless intrascleral fixation of secondary intraocular lenses in human eyes," Retina, vol. 38, no. 1, pp. S31-S40, 2018.

[12] Y. Matsui, H. Matsubara, T. Hanemoto, and M. Kondo, "Exposure of haptic of posterior chamber intraocular lens after sutureless intrascleral fixation," BMC Ophthalmology, vol. 15, no. 1, p. 104, 2015.

[13] A. M. Abbey, R. M. Hussain, A. R. Shah, L. J. Faia, J. D. Wolfe, and G. A. Williams, "Sutureless scleral fixation of intraocular lenses: outcomes of two approaches. the 2014 Yasuo Tano memorial lecture," Graefe's Archive for Clinical and Experimental Ophthalmology, vol. 253, no. 1, pp. 1-5, 2015.

[14] A. S. W. Luk, A. L. Young, and L. L. Cheng, "Long-term outcome of scleral-fixated intraocular lens implantation," British Journal of Ophthalmology, vol. 97, no. 10, pp. 13081311, 2013.

[15] B. J. Vote, P. Tranos, C. Bunce, D. G. Charteris, and L. Da Cruz, "Long-term outcome of combined pars plana vitrectomy and scleral fixated sutured posterior chamber intraocular lens implantation," American Journal of Ophthalmology, vol. 141, no. 2, pp. 308-312, 2006.

[16] A. S. McAllister and L. W. Hirst, "Visual outcomes and complications of scleral-fixated posterior chamber intraocular lenses," Journal of Cataract and Refractive Surgery, vol. 37, no. 7, pp. 1263-1269, 2011.

[17] E. G. Buckley, "Safety of transscleral-sutured intraocular lenses in children," Journal of American Association for Pediatric Ophthalmology and Strabismus, vol. 12, no. 5, pp. 431-439, 2008.

[18] R. Asadi and A. Kheirkhah, "Long-term results of scleral fixation of posterior chamber intraocular lenses in children," Ophthalmology, vol. 115, no. 1, pp. 67-72, 2008.

[19] C.-S. Yang and Y.-J. Chao, "Long-term outcome of combined vitrectomy and transscleral suture fixation of posterior chamber intraocular lenses in the management of posteriorly dislocated lenses," Journal of the Chinese Medical Association, vol. 79, no. 8, pp. 450-455, 2016.

[20] S. J. Kim, S. J. Lee, C. H. Park, G. Y. Jung, and S. H. Park, "Long-term stability and visual outcomes of a single-piece, foldable, acrylic intraocular lens for scleral fixation," Retina, vol. 29, no. 1, pp. 91-97, 2009.

[21] G. M. Cavallini, V. Volante, M. De Maria et al., "Long-term analysis of IOL stability of the Lewis technique for scleral fixation," European Journal of Ophthalmology, vol. 25, no. 6, pp. 525-528, 2015.

[22] N. Kandemir Besek, N. Alagoz, G. Gumus et al., "Long-term results of aphakia management by scleral fixation intraocular lens placement with knotless transscleral $Z$-suture method," International Ophthalmology, vol. 40, no. 6, pp. 1449-1454, 2020.

[23] S. Dimopoulos, V. Dimopoulos, G. Blumenstock et al., "Longterm outcome of scleral-fixated posterior chamber intraocular lens implantation with the knotless $Z$-suture technique," Journal of Cataract and Refractive Surgery, vol. 44, no. 2, pp. 182-185, 2018.

[24] H. Zhao, W. Wang, Z. Hu, and B. Chen, "Long-term outcome of scleral-fixated intraocular lens implantation without conjunctival peritomies and sclerotomy in ocular trauma patients," BMC Ophthalmology, vol. 19, no. 1, p. 164, 2019.

[25] D. Lockington, N. Q. Ali, R. Al-Taie, D. V. Patel, and C. N. J. McGhee, "Outcomes of scleral-sutured conventional and aniridia intraocular lens implantation performed in a university hospital setting," Journal of Cataract and Refractive Surgery, vol. 40, no. 4, pp. 609-617, 2014.

[26] G. Bading, J. Hillenkamp, H. G. Sachs, V.-P. Gabel, and C. Framme, "Long-term safety and functional outcome of combined pars plana vitrectomy and scleral-fixated sutured posterior chamber lens implantation," American Journal of Ophthalmology, vol. 144, no. 3, pp. 371-377, 2007.

[27] L. Krause, N. E. Bechrakis, H. Heimann, S. Salditt, and M. H. Foerster, "Implantation of scleral fixated sutured posterior chamber lenses: a retrospective analysis of 119 cases," International Ophthalmology, vol. 29, no. 4, pp. 207-212, 2009.

[28] G. T. Kokame, R. T. Yanagihara, J. G. Shantha, and K. N. Kaneko, "Long-term outcome of pars plana vitrectomy and sutured scleral-fixated posterior chamber intraocular lens implantation or repositioning," American Journal of Ophthalmology, vol. 189, pp. 10-16, 2018.

[29] G. Rogers, H. Mustak, M. Hann, D. Steven, and C. Cook, "Sutured posterior chamber intraocular lenses for traumatic cataract in Africa," Journal of Cataract and Refractive Surgery, vol. 40, no. 7, pp. 1097-1101, 2014. 
[30] R. Mannan, R. Sinha, N. Sharma, A. Pruthi, J. S. Titiyal, and R. B. Vajpayee, "Intrascleral reverse pocket approach of transconjunctival transscleral sulcus fixation of intraocular lens in eyes with ocular trauma," Eye \& Contact Lens: Science \& Clinical Practice, vol. 37, no. 5, pp. 316-319, 2011.

[31] D. Bhojwani, A. R. Vasavada, V. Vasavada, S. Vasavada, M. R. Praveen, and S. Srivastava, "Intraoperative performance and long-term postoperative outcomes after scleral fixation of IOLs with polytetrafluoroethylene suture," Journal of Cataract and Refractive Surgery, vol. 46, no. 11, pp. 1480-1486, 2020.

[32] E. Wasiluk, P. Krasnicki, D. A. Dmuchowska, E. ProniewskaSkrętek, and Z. Mariak, "The implantation of the scleralfixated posterior chamber intraocular lens with $9 / 0$ polypropylene sutures-long-term visual outcomes and complications," Advances in Medical Sciences, vol. 64, no. 1, pp. 100-103, 2019.

[33] K. Schulze-Bonsel, N. Feltgen, H. Burau, L. Hansen, and M. Bach, "Visual acuities "hand motion" and "counting fingers" can be quantified with the freiburg visual acuity test," Investigative Opthalmology \& Visual Science, vol. 47, no. 3, pp. 1236-1240, 2006.

[34] E. S. Malbran, E. Malbran Jr., and I. Negri, "Lens guide suture for transport and fixation in secondary IOL implantation after intracapsular extraction," International Ophthalmology, vol. 9, no. 2-3, pp. 151-60, 1986.

[35] L. Yeung, N.-K. Wang, W.-C. Wu, and K.-J. Chen, "Combined 23-gauge transconjunctival vitrectomy and scleral fixation of intraocular lens without conjunctival dissection in managing lens complications," BMC Ophthalmology, vol. 18, no. 1, p. 108, 2018.

[36] P. C. Jacobi, T. S. Dietlein, and F. K. Jacobi, "Scleral fixation of secondary foldable multifocal intraocular lens implants in children and young adults," Ophthalmology, vol. 109, no. 12, pp. 2315-2324, 2002.

[37] Y. Chantarasorn, S. Techalertsuwan, P. Siripanthong, and A. Tamerug, "Reinforced scleral fixation of foldable intraocular lens by double sutures: comparison with intrascleral intraocular lens fixation," Japanese Journal of Ophthalmology, vol. 62, no. 3, pp. 365-372, 2018.

[38] Y. U. Shin, M. Seong, H. Y. Cho, and M. H. Kang, "Novel technique to overcome the nonavailability of a long needle 9-0 polypropylene suture for sutured scleral fixation of the posterior chamber intraocular lens using a single fisherman's knot," Journal of Ophthalmology, vol. 2017, Article ID 2683415, 4 pages, 2017.

[39] D. C. Terveen, N. R. Fram, B. Ayres, and J. P. Berdahl, "Smallincision 4-point scleral suture fixation of a foldable hydrophilic acrylic intraocular lens in the absence of capsule support," Journal of Cataract and Refractive Surgery, vol. 42, no. 2, pp. 211-216, 2016.

[40] A. Durak, H. F. Oner, N. Koçak, and S. Kaynak, "Tilt and decentration after primary and secondary transsclerally sutured posterior chamber intraocular lens implantation," Journal of Cataract and Refractive Surgery, vol. 27, no. 2, pp. 227-232, 2001. 